\title{
Parkinsonian Syndrome and Toxoplasmic Encephalitis
}

\author{
Anca-Adriana Arbune ${ }^{1 *}$, Manuela Arbune ${ }^{2}$, Victorita Stefanescu ${ }^{2}$ \\ ${ }^{1}$ Carol Davila Medicine and Pharmacy University, Bucharest, Romania \\ 2 "Dunarea de Jos" University Galati, Romania
}

\begin{abstract}
Toxoplasmosis encephalitis in patients with human immunodeficiency virus may progress rapidly with a potentially fatal outcome. Less common neurological symptoms associated with this are Parkinsonism, focal dystonia, rubral tremor and hemichorea-hemiballismus syndrome.

A 58 year old woman suddenly lost consciousness and was admitted to the emergency service. Her medical history was unremarkable, except for frequent headaches in the last year, recurrent herpes simplex skin lesions and an episode of urticaria. A computer tomography scan showed supra and infra-tentorial lesions on suggestive of cerebral toxoplasmosis. Both Toxoplasma gondii and HIV tests were positive. In the intensive care unit, antiparasitic and antiretroviral drugs were administered, and she recovered from the coma after six weeks but presented with tetraparesis, diplopia, and depression. The LCD4 count increased from 7 to $128 / \mathrm{mm} 3$. The neurological lesions slowly resolved over the next two months, although postural instability, rigidity, bradykinesia and predominantly left side tremor persisted. Mild improvement was achieved after the administration of levodopa.

Associated Parkinsonian syndrome in HIV patients is a rare condition, explained by the location of the brain and basal ganglia lesions, and by the observed effect of Toxoplasma gondii which increases dopamine metabolism in neural cells. Early HIV diagnostic and treatment are necessary to prevent neurological disability.
\end{abstract}

Keywords: parkinsonism, toxoplasmosis, AIDS, dopamine

Received: 10 January 2016 / Accepted: 26 March 2016

\section{INTRODUCTION}

The clinical outcome of toxoplasmosis is asymptomatic in over $80 \%$ of immunocompetent adults. Encephalitis with Toxoplasma gondii can be found in the immunosuppressed patient, as a neurologic disease related to acquired immunodeficiency syndrome (AIDS) [1]. The subacute clinical onset of focal signs is the most common presentation while the acute onset of seizures or cerebral haemorrhage is reported in $15-25 \%$ of cases [2]. A high mortality risk is associated with acute toxoplasmosis, characteristic of diffuse encephalitis, and insignificant computer topographic findings, except brain atrophy. Neurological lesions occur with cranial nerve disorders, disorientation, altered mental status, lethargy or coma [3]. Uncommon clinical presentations are mental illness such as paranoid psychosis, dementia, anxiety or agitation. Other less typical manifestations are Parkinsonism, focal dystonia, rubral tremor, hemichorea-hemiballismus, panhypopituitarism, diabetes insipidus or the syndrome of inappropriate antidiuretic hormone secretion [4].

\section{CASE REPORT}

A 58 year old woman suddenly lost consciousness before her usual bedtime. She was found by her family the next day when she was admitted to the emergency service.

Her family stated that she did not smoke or have a history of alcohol or recreational drugs misuse. Her personal medical history was unremarkable, except having had five elective abortions twenty years ago, frequent headache episodes in the last year, recurrent herpes simplex skin lesions and a single urticarial event.

A physical examination recorded a Glasgow coma score 5, tetraparesis, right convergent strabismus, no

\footnotetext{
Correspondence to: Manuela Arbune, Hospital of Infectious Diseases "Sf. Cuvioasa Parascheva" Galați, Address: Str. Traian, Nr. 393, Phone: 0236 334022, 0236 334032.

E-mail: arbunemanuela@yahoo.com

Manuela Arbune: "Dunarea de Jos" University Galati, Romania, Str. Domnească, nr. 47, 800008 - Galați, Romania

Victorita Stefanescu: "Dunarea de Jos" University Galati, Romania, Str. Domnească, nr. 47, 800008 - Galați, Romania
} 

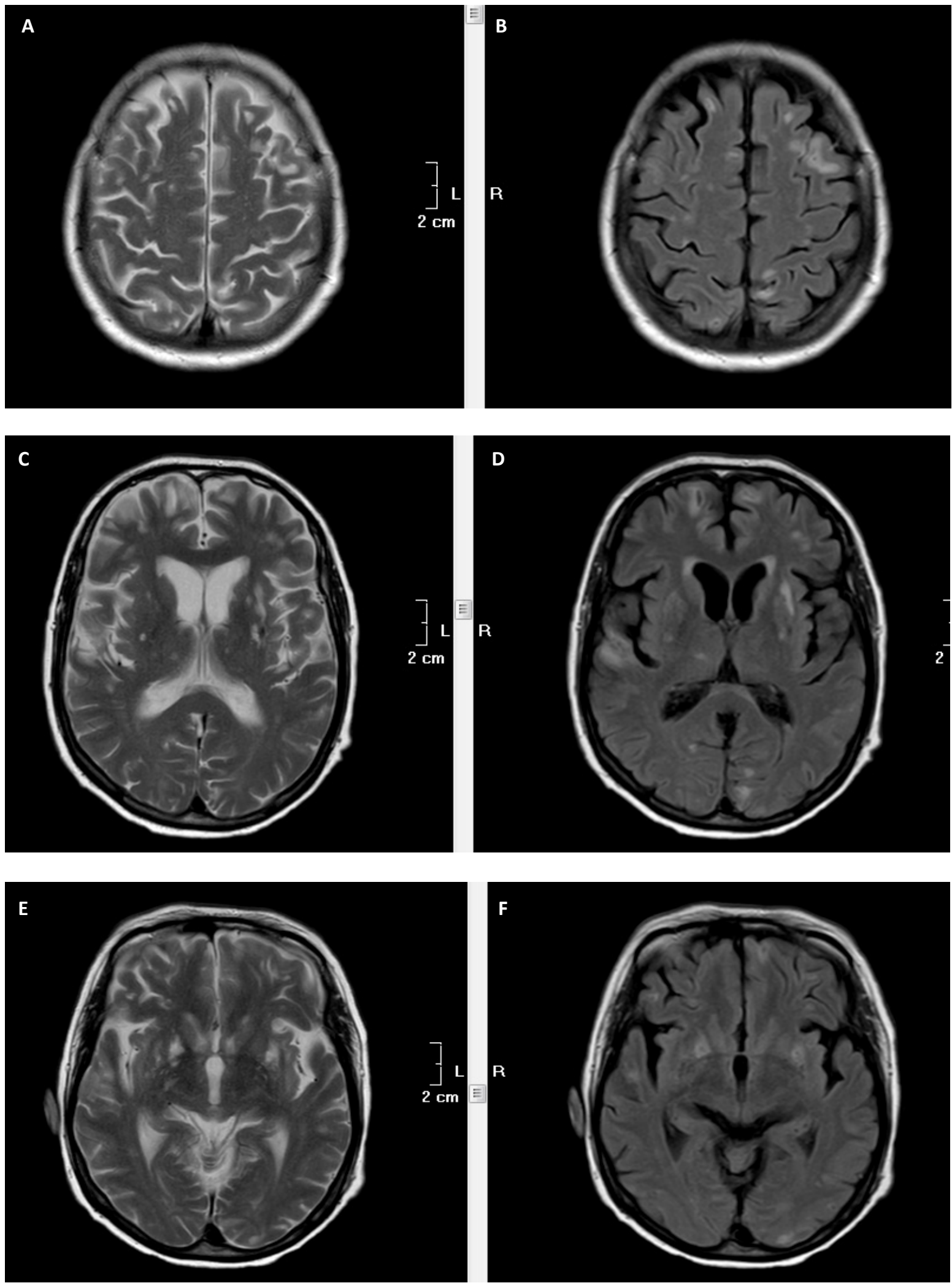

Fig. 1. Native cerebral MRI axial T2 section (A, C, E), FLAIR (B, D, F) at the same comparison level. There are identified multiple small round lesions hyper dense in T2 (liquid), inhomogeneous FLAIR signal (determined by haemorrhages or calcifications), situated supra and infra-tentorial, cortical, sub-cortical and in the basal ganglia, predominantly on the left side. 
nuchal stiffness, bilateral pulmonary rales, and persistent hypotension after fluid administration. A cerebral computer tomography scan revealed supra and infra-tentorial lesions that suggested cerebral toxoplasmosis. Polymerase chain reaction for Toxoplasma gondii in cerebrospinal fluid and human immunodeficiency virus (HIV) in blood, were positive. Baseline blood evaluation showed an LCD4 count of $7 / \mathrm{mm}^{3}$ and ARN-HIV 533000 copies/ml. Stage C3 by CDC HIV classification was confirmed. Antiparasitic drugs were administered in the intensive care unit, together with pyrimethamine, clindamycin, and trimethoprim-sulfamethoxazole, highly active antiretroviral treatment (HAART), lamivudine and lopinavir/ritonavir. She re- covered from the coma after six weeks, her neurological recovery was unusual, presenting as she did with tetraparesis, diplopia and depression. After six months she required support for walking and choreoathetotic movements appeared. Co-medication with levodopa, clonazepam and sertraline were initiated following the recommendation from neurologist.

Twelve months after the initial HIV diagnosis, her LCD 4 count increased to $224 / \mathrm{mm}^{3}$ with ARN-HIV $65 / \mathrm{mm}^{3}$. Neurological lesion slowly improved, although there was a persistence of postural instability, rigidity, bradykinesia and predominantly left side tremor. A mild improvement was achieved after levo-
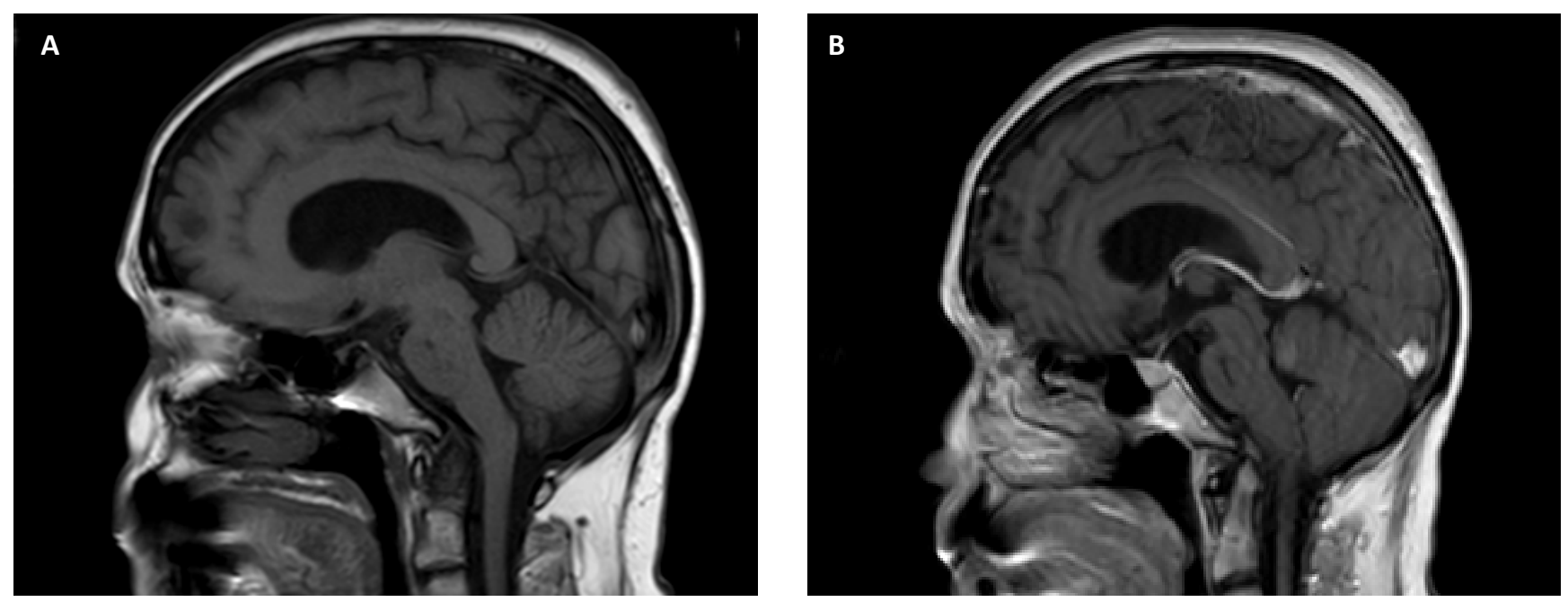

Fig. 2. Native sagittal T1 section (A), post contrast T1 (B), exhibit small lesions in the frontal region, with discrete peripheral contrast enhancement ("bullseye" images) and homogeneous cerebellar enhancement.
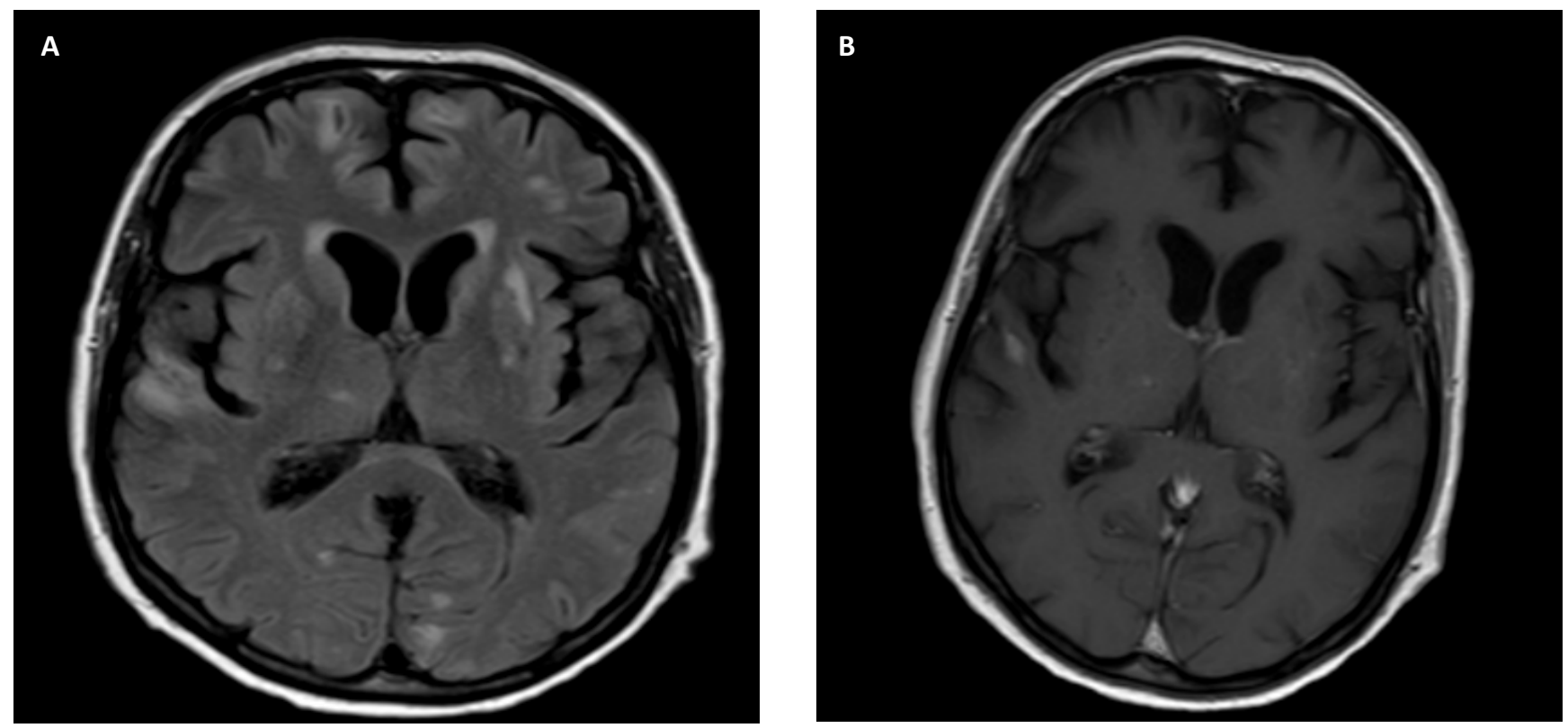

Fig. 3. Axial FLAIR section (A), post-contrast T1 (B), with reduced lesions after enhancement. 
dopa use while multiple nodular enhancing lesions localized in the subcortical frontoparietal region and basal ganglia were identified on magnetic resonance imaging (Figure 1-3).

\section{DISCUSSIONS}

Movement disorders have been reported since the first descriptions of neurological complications of AIDS, with $50 \%$ of patients reported, in subsequent prospective studies, to have developed extrapyramidal features [3]. The incidence of Parkinsonism in HIV patients was shown to diminish after HAART treatment, while the age at which Parkinsonian syndrome occurred, increased from 40 to 50 years old [5]. Advanced HIV infection is a contributing factor to the progression of Parkinson's disease, as well as other neurodegenerative diseases [6]. On the other hand, movement disorders in HIV patients are related either directly to HIV disease in the brain or secondary to opportunistic neurological diseases or drug-induced extrapyramidal syndrome [7].

Toxoplasmosis encephalitis is the most common neurologic opportunistic disease. Toxoplasma gondii is a parasite considered to be involved in several different neuropsychiatric disorders and causes increased dopamine metabolism in neural cells. Studies on animal models identified a tyrosine hydroxylase enzyme in the parasite genome, an enzyme that limits dopamine synthesis [8]. An increased incidence of toxoplasmosis seropositivity was found in patients with Parkinson's disease since dopamine is the biochemical substrate of this disorder $[9,10]$.

There are several observed mechanisms which could account for Parkinsonism in the patient. These include basal ganglia neuronal loss by HIV replication, the decrease of substantia nigra, direct destruction of substantia nigra by toxoplasma abscesses, interference of toxoplasma parasite with dopamine metabolism, cerebral vascular deficiency, possible neurotoxicity of antiretroviral drugs or the uncommon event of immune reconstruction syndrome.

\section{- CONCLUSIONS}

Late presentation with advanced HIV immunosuppression is associated with a high risk of opportunistic neurologic diseases. Associated Parkinsonian syndrome in HIV patients is a rare condition that is explained by the location of the brain lesions, including the basal ganglia, and the observed effect of Toxoplasma gondii. Movement disorders in HIV patients are related to complex pathological mechanisms, and early HIV diagnostic and treatment are necessary to prevent neurological disability in these cases.

\section{CONFLICT OF INTEREST}

Nothing to declare

\section{REFERENCES}

1. 1993 revised classification system for HIV infection and expanded surveillance case definition for AIDS among adolescents and adults. MMWR Recomm Rep. 1992;41(RR17):1-19.

2. Montoya JG, Liesenfeld O. Toxoplasmosis. Lancet. 2004;363: 1965-76.

3. Tse W, Cersosimo MG, Gracies JM, Morgello S, Olanow CW, Koller W. Movement disorders and AIDS: a review. Parkinsonism Relat Disord. 2004;10:323-34.

4. Cardoso F. HIV-related movement disorders: epidemiology, pathogenesis and management. CNS Drugs. 2002;16:663-8.

5. Rosso AL, Mattos JP, Correa RB, Nicaretta DH, Novis SA. Parkinsonism and AIDS: a clinical comparative study before and after HAART. Arq Neuropsiquiatr. 2009;67:827-30.

6. Brew BJ, Crowe SM, Landay A, et al. Neurodegeneration and ageing in the HAART era. J Neuroimmune Pharmacol. 2009;4:163-74.

7. Factor SA, Troche-Panetto M, Weaver SA. Dystonia in AIDS: report of four cases. Mov Disord. 2003;18:1492-8.

8. Prandovszky E, Gaskell E, Martin H, Dubey JP, Webster JP, et al. The neurotropic parasite Toxoplasma gondii increases dopamine metabolism. PLoS ONE. 2011;6(9):e23866.

9. Miman O, Kusbeci OY, Aktepe OC, Cetinkaya Z. The probable relation between Toxoplasma gondii and Parkinson's disease. Neurosci Lett. 2010;475:129-31.

10. Szewczyk-Krolikowski K, Tomlinson P, Nithi K, et al. The influence of age and gender on motor and non-motor features of early Parkinson's disease: initial findings from the Oxford Parkinson Disease Center (OPDC) discovery cohort. Parkinsonism Relat Disord. 2014;20:99-105. 